\title{
Splitting Theorems for Spatially Closed Space-Times
}

\author{
Gregory J. Galloway \\ Department of Mathematics, University of Miami, Coral Gables, FL 33124, USA
}

\begin{abstract}
A Lorentzian splitting theorem is obtained for spatially closed spacetimes. The proof employs and extends some recent results of Bartnik and Gerhardt concerning the existence and rigid uniqueness of compact maximal hypersurfaces in spatially closed space-times. A splitting theorem for spatially closed time-periodic space-times, which generalizes a result first considered by Avez, is derived as a corollary.
\end{abstract}

\section{Introduction}

Yau [12] has posed the problem of establishing a Lorentzian splitting theorem analogous to the splitting theorem of Cheeger and Gromoll [5] for Riemannian manifolds. In this paper we prove the following splitting result for spatially closed space-times.

Theorem 1.1. Let $V$ be a space-time which has the following properties:

(A) $V$ contains a compact Cauchy surface.

(B) $V$ satisfies the timelike convergence condition, i.e., $\operatorname{Ric}(X, X) \geqq 0$ for all timelike $X$.

(C) $V$ contains a timelike curve which is future and past complete.

(D) For each $p \in V$, every future (past) inextendible null geodesic $\eta$ issuing from $p$ reaches a point in the timelike future (past) of $p$, i.e., $\eta \cap I^{+}(p) \neq \phi\left(\eta \cap I^{-}(p) \neq \phi\right)$.

Then $V$ splits into the pseudo-Riemannian product of $\left(\mathbb{R},-d t^{2}\right)$ and $(M, h)$, where $M$ is a smooth compact spacelike hypersurface and $h$ is the induced metric on $M$. In particular if $V$ is Ricci flat and $\operatorname{dim} V=4$ then $V$ is flat.

Remarks. We shall always use the term "hypersurface" to mean "hypersurface without boundary." Put more succinctly, condition (D) states that there exists a null cut point along each future and past inextendible null geodesic. In Sect. 3 it is shown that for space-times admitting a compact Cauchy surface, (D) is equivalent to the requirement that there be no observer with a nontrivial future or past event horizon. 
Theorem 1.1 can be viewed as a singularity theorem of sorts: If $V$ is a space-time satisfying (A) and (B) which does not split in the sense described above (e.g. is not static) then either (C) fails to hold, in which case $V$ is singular in the usual sense, or (D) fails to hold, in which case one suspects the occurrence of a singularity. Interpreted in this way, Theorem 1.1 is an example within singularity theory of a rigidity result in the sense described in Cheeger and Ebin [4].

The proof of Theorem 1.1 rests heavily on different aspects of the important recent papers of Bartnik [2] and Gerhardt [7]. Gerhardt ([7], Theorem 7.4) has proved a splitting result for the region between two compact maximal hypersurfaces in a globally hyperbolic space-time satisfying the timelike convergence condition. In order to use this result to prove Theorem 1.1 it will be necessary to establish new criteria for the existence of compact maximal hypersurfaces. By using singularity theory in an essential way we are able to modify an existence proof of Bartnik ([2], Theorem 4.1) to establish the existence of compact maximal hypersurfaces in space-times satisfying (A)-(C) and a compactness condition implied by (D). This existence result is presented in Sect. 2. In Sect. 3 we obtain a number of equivalent characterizations of condition (D) and present the proof of Theorem 1.1.

In Sect. 4 we derive as a corollary to Theorem 1.1 the following rigid version of Tipler's [11] No Return Theorem, which settles a problem dating back to the 1963 paper of Avez [1].

Theorem 1.2. Suppose $V$ is a spatially closed time-periodic space-time which satisfies the timelike convergence condition. Then the conclusions of Theorem 1.1 hold.

Avez had considered the Ricci flat case, but his proof contains a well-known error (see Marsden and Tipler [10]).

In order to simplify some statements occurring in the following sections, we shall refer to the hypotheses of Theorem 1.1 by letter only. Frequent use is made of the causal theory of space-time, for which Hawking and Ellis [9] is a standard reference.

\section{Existence of Compact Maximal Hypersurfaces}

Theorem 2.1. Let $V$ be a space-time satisfying $(A),(B)$, and $(C)$. Let $S$ be a smooth compact spacelike hypersurface in $V$ such that the collection of compact spacelike hypersurfaces meeting $S$ stays in a compact subset of $V$. Then $V$ contains a smooth compact maximal hypersurface which meets $S$.

The proof is a modification of Theorem 4.1 in Bartnik [2]. The essential new ingredient is the application of singularity theory, for which one needs the timelike convergence condition. In fact Theorem 2.1 is false without this assumption.

We shall isolate in terms of a lemma precisely what is needed from Bartnik's proof. First, let us introduce some notation. Let $t: V \rightarrow \mathbb{R}$ be a smooth time function all of whose level surfaces are Cauchy. Let $S_{0}$ be the slice $t=0$. $V$ is homeomorphic to $\mathbb{R} \times S_{0}$ via the correspondence $p \leftrightarrow(t(p), \pi(p))$, where $\pi: V \rightarrow S$ is projection along the flowlines of $\nabla t$ into $S_{0}$. For $t_{1}, t_{2} \in \mathbb{R}$, let

$$
\mathscr{C}=\left\{u \in C^{2}\left(S_{0}\right): \text { graph } u \text { is spacelike and } t_{1} \leqq u \leqq t_{2}\right\},
$$


where graph $u \equiv\left\{(u(x), x): x \in S_{0}\right\}$. Let $H(u)=$ mean curvature of graph $u$, and $H^{0}(u)(x)=$ mean curvature of the slice $t=u(x)$ at the point $(u(x), x)$. (We use the sign convention in which $H>0$ corresponds to expansion on the average of the future pointing normal.)

Lemma (Bartnik [2]). Let $t: V \rightarrow \mathbb{R}$ be a smooth time function such that the slice $S_{0}$ is compact. Fix $\varepsilon>0$ and $t_{1}<0<t_{2}$. If for each $\sigma, 0<\sigma<1, u \in \mathscr{C}$ satisfies the strict inequality

$$
t_{1}<u<t_{2}
$$

whenever u satisfies,

$$
H(u)=(1-\sigma) v(u) H^{0}(u)+\varepsilon u,
$$

where $v(u)$ is defined as in [2], then there exists $u_{\varepsilon} \in C^{\infty}\left(S_{0}\right)$ such that graph $u_{\varepsilon}$ is spacelike and has mean curvature $H\left(u_{\varepsilon}\right)=\varepsilon u_{\varepsilon}$.

Proof of Theorem 2.1. Choose a smooth time function $t: V \rightarrow \mathbb{R}$ whose level surfaces are compact Cauchy surfaces. By Budic et al. [3] $S$ is Cauchy and hence can be represented as a graph over the slice $t=0$. By Proposition 3.2 in Bartnik [2] one can choose the time function so that $S$ corresponds to the slice $t=0$. Furthermore by rescaling the time function one can choose times $\ldots t_{n-1}<t_{n}<t_{n+1} \ldots$ such that $t_{0}=0, t_{n} \rightarrow \pm \infty$ as $n \rightarrow \pm \infty$ and for $n \neq 0$,

$$
\left|t_{n}\right|>|n| \lambda_{n}
$$

where $\lambda_{n}=\sup \left|H_{n}\right|$ and $H_{n}$ is the mean curvature of the slice $t=t_{n}$.

For each positive integer $n$ define,

$$
\mathscr{C}_{n}=\left\{u \in C^{2}(S): \text { graph } u \text { is spacelike and } t_{-n} \leqq u \leqq t_{n}\right\} .
$$

Suppose for some $\sigma, 0<\sigma<1, u \in \mathscr{C}_{n}$ obeys

$$
H(u)=(1-\sigma) v(u) H^{0}(u)+n^{-1} u .
$$

At points where $\nabla u=0, v(u)=1$. Thus, if $u=t_{n}$ at its maximum, then the equation above becomes, $H(u)=(1-\sigma) H_{n}+n^{-1} t_{n}$.

Hence,

$$
H(u)-H_{n}=n^{-1}\left(t_{n}-n \sigma H_{n}\right) \geqq n^{-1}\left(t_{n}-n \lambda_{n}\right)>0,
$$

where the last inequality follows from (2.1). However, since graph $u$ lies below the slice $t=t_{n}$ we must have $H(u) \leqq H_{n}$ at any point of tangency. Arguing similarly at a minimum of $u$ shows that $u$ obeys the strict inequalities $t_{-n}<u<t_{n}$. Thus, by the lemma there exists $u_{n} \in C^{\infty}(S)$ with mean curvature,

$$
H\left(u_{n}\right)=n^{-1} u_{n} \text {. }
$$

Suppose the family $\left\{u_{n}\right\}_{n=1}^{\infty}$ obeyed a uniform height estimate independent of $n$. Then by the gradient estimate in Gerhardt ([7], Theorem 4.1; see also Theorem 3.1 in [2]) and standard estimates for linear and quasilinear elliptic equations (see Gilbarg and Trudinger [8]), the family would be uniformly bounded in $C^{2, \lambda}$-norm. Hence, one could extract a subsequence $\left\{u_{n_{k}}\right\}$ converging to $u \in C^{2}(S)$ with mean curvature zero. By standard regularity results $u$ would be $C^{\infty}$. 
The desired height estimate can be obtained using singularity theory. By the compactness assumption it suffices to show that graph $u_{n}$ meets $S$ for all $n$. If graph $u_{n}$ does not meet $S$ then by (2.2) the mean curvature of graph $u_{n}$ is either everywhere strictly positive or everywhere strictly negative. Suppose $H\left(u_{n}\right)<0$. Since we are working in a globally hyperbolic space-time, standard singularity theory (see e.g. Hawking and Ellis [9]) implies that every future inextendible timelike curve issuing from graph $u_{n}$ has finite length. But this contradicts assumption (C).

Finally, since graph $u_{n}$ meets $S$ for all $n$, the limit surface of mean curvature zero must also meet $S$.

\section{Proof of Theorem 1.1}

We begin this section by establishing a number of equivalent characterizations of condition (D).

Theorem 3.1. Let $V$ be a space-time which contains a compact Cauchy surface. Then the following conditions are equivalent:

(D 1) Condition (D) holds.

(D2) For each compact set $K \subset V$, the collection of achronal subsets of $V$ which meet $K$ is contained in a compact subset of $V$.

(D 3) All closed achronal subsets of V are compact.

(D4) $\partial I^{+}(p), \partial I^{-}(p)$ are compact for all $p \in V$.

(D 5) For each inextendible timelike curve $\gamma, I^{+}(\gamma)=I^{-}(\gamma)=V$, i.e., there are no observer horizons.

Proof. (D 1) $\Rightarrow$ (D 2): Let $W^{+}=\bigcup_{p \in K} E^{+}(p)$, where $E^{+}(p) \equiv J^{+}(p)-I^{+}(p)=\partial I^{+}(p)$, since $V$ is globally hyperbolic. We prove that $W^{+}$is compact. Let $T$ be a smooth future pointing unit timelike vector field on $V$. Define,

$\mathscr{N}=\{(p, N): N$ is a null vector at $p \in K$ such that $\langle N, T\rangle=-1\}$.

$\mathcal{N}$ is a compact subset of the null vector bundle over $V$.

We prove sequential compactness. Let $\left\{q_{k}\right\}$ be a sequence in $W^{+}$. Then $q_{k}=\exp _{p_{k}} s_{k} N_{k}$ for some $\left(p_{k}, N_{k}\right) \in \mathcal{N}$. There is a subsequence, again denoted by $\left\{\left(p_{k}, N_{k}\right)\right\}$, such that $\left(p_{k}, N_{k}\right) \rightarrow(p, N) \in \mathscr{N}$. Let $\eta:[0, a) \rightarrow V$ be the inextendible null geodesic defined by: $\eta(s)=\exp _{p} s N$.

We claim that $\bar{s} \equiv \lim \sup _{k}<a$. Indeed, suppose $\bar{s} \geqq a$. Then, by taking a subsequence, we can assume $s_{k} \uparrow \bar{s} \geqq a$. By assumption $p \ll \eta\left(s_{0}\right)$ for some $s_{0} \in(0, a)$. Consider the null geodesic $\eta_{k}$ defined by: $\eta_{k}(s)=\exp _{p_{k}} s N_{k}$. Since $s_{0}<\bar{s}, \eta_{k}\left(s_{0}\right)$ is defined for all $k$ sufficiently large. Since $p_{k} \rightarrow p$ and $\eta_{k}\left(s_{0}\right) \rightarrow \eta\left(s_{0}\right)$, we have for $k$ large, $p_{k} \ll \eta_{k}\left(s_{0}\right)<\eta_{k}\left(s_{k}\right)=q_{k}$, which contradicts $q_{k} \in E^{+}\left(p_{k}\right)$.

Hence, $\bar{s}=\lim \sup s_{k}<a$. Let $q=\eta(\bar{s})$. Clearly $q \in E^{+}(p)$, otherwise $q_{k} \in I^{+}\left(p_{k}\right)$ for some $k$. Thus $\left\{q_{k}\right\}$ has a subsequence converging to $q \in W^{+}$. Therefore, $W^{+}$and similarly $W^{-}$, which is defined time-dually, are compact.

Let $S$ be any achronal subset of $V$ which meets $K$. To complete the proof we show that $S \subset J^{+}\left(W^{-}\right) \cap J^{-}\left(W^{+}\right)$. Let $p \in S \cap K$. The argument showing that $W^{+}$is compact implies in particular that $E^{+}(p)$ is compact. Standard arguments in 
causal theory can be used to show that $E^{+}(p)$ is an achronal Cauchy surface, but this fact follows, in particular, from Corollary 1 in [6]. Hence, for each $q \in S$ either $q \in I^{+}\left(E^{+}(p)\right)$ or $q \in J^{-}\left(E^{+}(p)\right)$. However the former case violates the achronality of $S$. Thus, $S \subset J^{-}\left(E^{+}(p)\right) \subset J^{-}\left(W^{+}\right)$. One shows similarly that $S \subset J^{+}\left(W^{-}\right)$.

(D 2) $\Rightarrow$ (D 3): This follows immediately.

(D 3) $\Rightarrow$ (D 4): The sets $\partial I^{ \pm}(p)$ are closed and achronal.

(D 4) $\Rightarrow$ (D 5): Let $\gamma$ be an inextendible timelike curve in $V$. For each $q \in V$, $E^{-}(q)$ is compact and hence, as discussed above, is an achronal Cauchy surface. Thus $\gamma$ meets $E^{-}(q)$ and hence $q \in I^{+}(\gamma)$. Therefore $I^{+}(\gamma)=V$ and similarly $I^{-}(\gamma)=V$.

(D 5) $\Rightarrow$ (D 1): Suppose (D) does not hold. Then, without loss of generality, there exists a future inextendible null geodesic $\eta$ issuing from some point $p$ such that $I^{-}(\eta) \cap \eta=\phi$, and hence $I^{-}(\eta) \neq V$. One easily constructs an inextendible timelike curve $\gamma$ such that $\gamma \subset I^{-}(\eta)$. But then $I^{-}(\gamma) \neq V$, contradicting (D 5).

The proof of Theorem 1.1 employs the splitting result of Gerhardt [7, Theorem 7.4] mentioned in the introduction which we now discuss in more detail. Given a smooth spacelike hypersurface $M \subset V$ we can introduce the associated normal exponential map $\Phi$ defined by, $\Phi(\tau, p)=\exp _{p} \tau N$, where $N$ is the future directed unit normal to $M$.

Theorem (Gerhardt [7]). Let $V$ be a space-time satisfying (A) and (B). Let $M_{1}$ and $M_{2}$ be compact maximal hypersurfaces in $V$ with $d\left(M_{1}, M_{2}\right)=\delta>0(d=$ Lorentzian distance function). Suppose that the collection of compact spacelike hypersurfaces meeting $J^{+}\left(M_{1}\right) \cap J^{-}\left(M_{2}\right)$ remains in a compact subset of $V$. Then $M_{1} \subset I^{-}\left(M_{2}\right)$ and the normal exponential map $\Phi:[0, \delta] \times M_{1} \rightarrow J^{+}\left(M_{1}\right) \cap J^{-}\left(M_{2}\right)$ is an isometry (where it is understood that $[0, \delta] \times M_{1}$ carries the Lorentzian product metric $-d \tau^{2} \oplus h, h=$ induced metric on $M_{1}$ ).

We now proceed to the

Proof of Theorem 1.1. Let $\left\{S_{n}: n \in \mathbb{Z}\right\}$ be a family of compact slices of some smooth time function such that $S_{n} \subset I^{-}\left(S_{n+1}\right)$ for all $n$ and $V=\bigcup_{n \in \mathbb{Z}} J^{+}\left(S_{n}\right) \cap J^{-}\left(S_{n+1}\right)$. In view of Theorem 3.1 ((D) $\Rightarrow$ (D 2)) all the hypotheses of Theorem 2.1 (with $S=S_{n}$ ) are satisfied. Thus for each $n \in \mathbb{Z}$, there exists a smooth compact maximal hypersurface $M_{n}$ which meets $S_{n}$. By choosing the $S_{n}$ 's sufficiently far apart one can ensure that $d\left(M_{n}, M_{n+1}\right)=\delta_{n}>0$ for all $n$. Again, since (D) $\Rightarrow$ (D 2) all the hypotheses of Gerhardt's splitting result are satisfied. Thus, for each $n, M_{n}$ $C I^{-}\left(M_{n+1}\right)$ and the normal exponential map $\Phi_{n}:\left[0, \delta_{n}\right] \times M_{n} \rightarrow J^{+}\left(M_{n}\right)$ $\cap J^{-}\left(M_{n+1}\right)$ is an isometry. Furthermore, $\bigcup_{n \in \mathbb{Z}} J^{+}\left(M_{n}\right) \cap J^{-}\left(M_{n+1}\right)=V$ (as follows from (D) or (D 2)). It is then not difficult to see that the isometries $\Phi_{n}$ fit together so that the normal exponential map $\Phi_{0}:(a, b) \times M_{0} \rightarrow V$ (where $a=-\sum_{n=1}^{\infty} \delta_{-n}$ and $\left.b=\sum_{n=0}^{\infty} \delta_{n}\right)$ is an isometry. Finally, (C) implies that $a=-\infty$ and $b=\infty$, which completes the proof. 


\section{Spatially Closed Time-Periodic Space-Times}

There have been several definitions of SCTP (spatially closed time-periodic) spacetimes to appear in the literature. We adopt the following definition.

Definition 4.1. A space-time $V$ is SCTP if

(1) $V$ contains a compact spacelike Cauchy surface $S$,

(2) there exists a discrete group of isometries $\psi_{n}: V \rightarrow V, n \in \mathbb{Z}$, such that $S_{n}$ $\subset I^{-}\left(S_{n+1}\right)$ and $V=\bigcup_{n \in \mathbb{Z}} J^{+}\left(S_{n}\right) \cap J^{-}\left(S_{n+1}\right)$, where $S_{n}=\psi_{n}(S)$, and

(3) for each $p \in S$ there exists a positive integer $n$ such that $p \ll \psi_{n}(p)$.

The definition of a SCTP space-time given in Tipler [11] essentially requires only that (1) and (2) hold. A space-time which is SCTP in this sense can have some undesirable features. For example one can construct space-times which satisfy (1) and (2), but which do not satisfy (D) and do not contain any compact maximal hypersurfaces. The definitions of a SCTP space-time given in Avez [1] and Marsden and Tipler [10] require that the discrete isometry group arise as a subgroup of a timelike $\mathbb{R}$-action, i.e., an $\mathbb{R}$-action whose orbits are timelike curves. Condition (3) is then automatically satisfied.

The significance of condition (3) within the context of the present paper is described in the following theorem.

Theorem 4.1. Let V be a space-time satisfying conditions (1) and (2) of Definition 4.1. Then condition (3) holds if and only if (D) holds.

Proof. First suppose that (3) holds. Let $\eta$ be a future inextendible null geodesic issuing from a point $q$. By sliding $\eta$ via the isometries we may assume that $q \in J^{-}\left(S_{0}\right)$. For $n \geqq 0$, let $q_{n} \in \eta \cap S_{n}$, and consider the sequence $\left\{p_{n}\right\} \subset S_{0}$, where $p_{n}=\psi_{-n}\left(q_{n}\right)$. Let $p \in S_{0}$ be a limit point of $\left\{p_{n}\right\}$. By condition (3), there exists an integer $N>0$ such that $p \ll \psi_{N}(p)$. By passing to the subgroup of $\left\{\psi_{n}\right\}$ generated by $\psi_{N}$ we can assume without harm that $N=1$. Thus, there exists a neighborhood $U$ $C S_{0}$ of $p$ such that $U \ll \psi_{1}(U)$. (Here we are using the notation $A \ll B$ to mean that every point in $A$ can be joined to every point in $B$ by a future directed timelike curve.) Since isometries preserve causal relations we have

$$
\psi_{m}(U) \ll \psi_{n}(U) \text { for all } m, n \in \mathbb{Z}, m<n .
$$

Choose $p_{m}, p_{n} \in U$ with $0 \leqq m<n$. Then by (4.1), $q_{m}=\psi_{m}\left(p_{m}\right) \ll \psi_{n}\left(p_{n}\right)=q_{n}$. Thus, $q \ll q_{n}$, as required.

Now assume (D) ( $\Leftrightarrow$ (D 3); see Theorem 3.1) holds. If condition (1) fails for some $p \in S$, then $\left\{\psi_{n}(p): n \geqq 0\right\}$ is a closed achronal subset of $V$. Hence, by (D 3), this set is compact, which is impossible.

We mention the following corollary which is relevant to the work of Marsden and Tipler [10], but which will not be needed in the proof of Theorem 1.2.

Corollary. If $V$ is SCTP then V obeys the Marsden-Tipler avoidance of singularities condition. In fact, there exist Cauchy surfaces $S^{-}$and $S^{+}$, with $S^{-} \subset I^{-}\left(S^{+}\right)$, such that for any achronal set $A \subset V$ there exists a set $A^{\prime} C V$ isometric to $A$ (under one of the isometries $\left.\psi_{n}\right)$ which is contained in $J^{+}\left(S^{-}\right) \cap J^{-}\left(S^{+}\right)$. 
Proof. Let $K=J^{+}\left(S_{0}\right) \cap J^{-}\left(S_{1}\right)$. It follows from (D2) that there exist compact Cauchy surfaces $S^{-}, S^{+}$, with $S^{-} \subset I^{-}\left(S^{+}\right)$such that if $A^{\prime}$ is an achronal subset of $V$ which meets $K$ then $A^{\prime} \subset J^{+}\left(S^{-}\right) \cap J^{-}\left(S^{+}\right)$. The proof is completed by noting that any achronal set $A \subset V$ can be moved via the isometries $\psi_{n}$ to obtain an isometric set $A^{\prime}$ which meets $K$.

We conclude the paper with the proof of Theorem 1.2.

Proof of Theorem 1.2. It suffices to show that if $V$ is SCTP then conditions (A)-(D) hold. (A) and (B) hold by assumption. (D) holds by Theorem 4.1. That (C) holds can be seen as follows: Consider the quotient manifold $\tilde{V}=V /\left\{\psi_{n}\right\} . \tilde{V}$ is a compact space-time which carries a Lorentzian metric with respect to which the natural projection map is a local isometry. Since $\tilde{V}$ is compact it contains a closed timelike curve $\tilde{\gamma}$. By repeating loops we can view $\tilde{\gamma}$ as an inextendible curve which is future and past complete. Then by lifting $\tilde{\gamma}$ we obtain a future and past complete timelike curve in $V$.

Acknowledgements. I would like to thank Richard Hamilton for several informative conversations and Steve Harris for a correspondence concerning time-periodic space-times. I am especially grateful to Robert Bartnik for the interest he has taken in this work, his many valuable comments on an earlier version of this paper, and a suggestion for improving the statement of a preliminary version of Theorem 1.1. This work was carried out while I was visiting the University of California, San Diego.

\section{References}

1. Avez, A.: Essais de géometrie riemanniene hyperbolique globale. Applications à la rélativité generale. Ann. Inst. Fourier 13, 105-190 (1963)

2. Bartnik, R.: Existence of maximal hypersurfaces in asymptotically flat space-times. Commun. Math. Phys. 94, 155-175 (1984)

3. Budic, R., Isenberg, J., Lindblom, L., Yasskin, P.B.: On the determination of Cauchy surfaces from intrinsic properties. Commun. Math. Phys. 61, 87-95 (1978)

4. Cheeger, J., Ebin, D.: Comparison theorems in Riemannian geometry. Amsterdam: NorthHolland 1975

5. Cheeger, J., Gromoll, D.: The splitting theorem for manifolds of nonnegative Ricci curvature. J. Diff. Geom. 6, 119-128 (1971)

6. Galloway, G.: Some results on Cauchy surface criteria in Lorentzian geometry. in Ill. J. Math. (to appear)

7. Gerhardt, C.: $H$-surfaces in Lorentzian manifolds. Commun. Math. Phys. 89, 523-553 (1983)

8. Gilbarg, D., Trudinger, N.: Elliptic partial differential equations of second order. Berlin, Heidelberg, New York: Springer 1977

9. Hawking, S.W., Ellis, G.F.R.: The large scale structure of the universe. Cambridge: Cambridge University Press 1973

10. Marsden, J.E., Tipler, F.J.: Maximal hypersurfaces and foliation of constant mean curvature in general relativity. Phys. Rep. 66, 109-139 (1980)

11. Tipler, F.J.: General relativity and the eternal return in: Essays in general relativity. A Festschrift for Abraham H. Taub, ed. F. Tipler. New York: Academic Press 1980

12. Yau, S.-T., Problem section. In: Seminar in differential geometry, Yau, S.-T. (ed.). Ann. Math. Stud., Vol. 102. Princeton, NJ: Princeton University Press 1982

Communicated by S.-T. Yau

Received March 6, 1984; in revised form June 28, 1984 
